\title{
Stability of multistage stochastic programs
}

\author{
H. Heitsch ${ }^{1}$, W. Römisch ${ }^{1}$ and C. Strugarek ${ }^{2}$ \\ ${ }^{1}$ Humboldt-University Berlin, Institute of Mathematics, D-10099 Berlin, Germany \\ ${ }^{2}$ EdF R\&D, OSIRIS, 1 Avenue du Général de Gaulle F-92141 Clamart Cedex, France, and \\ Ecole Nationale des Ponts et Chaussées, and \\ Ecole Nationale Supérieure de Techniques Avancées
}

\begin{abstract}
Quantitative stability of linear multistage stochastic programs is studied. It is shown that the infima of such programs behave (locally) Lipschitz continuous with respect to the sum of an $L_{r}$-distance and of a distance measure for the filtrations of the original and approximate stochastic (input) processes. Various issues of the result are discussed and an illustrative example is given. Consequences for the reduction of scenario trees are also discussed.
\end{abstract}

Key Words: Stochastic programming, multistage, nonanticipativity, stability, filtration, probability metrics.

2000 MSC: $90 \mathrm{C} 15$

\section{Introduction}

We consider a finite horizon sequential decision process under uncertainty, in which a decision made at $t$ is based only on information available at $t(1 \leq t \leq T)$. We assume that the information is given by a discrete time multivariate stochastic process $\left\{\xi_{t}\right\}_{t=1}^{T}$ defined on some probability space $(\Omega, \mathcal{F}, \mathbb{P})$ and with $\xi_{t}$ taking values in $\mathbb{R}^{d}$. The information available at $t$ consists of the random vector $\xi^{t}:=\left(\xi_{1}, \ldots, \xi_{t}\right)$, and the stochastic decision $x_{t}$ at $t$ varying in $\mathbb{R}^{m_{t}}$ is assumed to depend only on $\xi^{t}$. The latter property is called nonanticipativity and is equivalent to the measurability of $x_{t}$ with respect to the $\sigma$-field $\mathcal{F}_{t} \subseteq \mathcal{F}$, which is generated by $\xi^{t}$. Hence, we have $\mathcal{F}_{t} \subseteq \mathcal{F}_{t+1}$ for $t=1, \ldots, T-1$ and we assume that $\mathcal{F}_{1}=\{\emptyset, \Omega\}$, i.e., $\xi_{1}$ and $x_{1}$ are deterministic and, with no loss of generality, that $\mathcal{F}_{T}=\mathcal{F}$.

More precisely, we consider the following linear multistage stochastic program

$$
\min \left\{\mathbb{E}\left[\sum_{t=1}^{T}\left\langle b_{t}\left(\xi_{t}\right), x_{t}\right\rangle\right] \mid \begin{array}{l}
x_{t} \in X_{t}, \\
x_{t} \text { is } \mathcal{F}_{t}-\text { measurable }, t=1, \ldots, T, \\
A_{t, 0} x_{t}+A_{t, 1} x_{t-1}=h_{t}\left(\xi_{t}\right), t=2, \ldots, T
\end{array}\right\},
$$

where the subsets $X_{t}$ of $\mathbb{R}^{m_{t}}$ are polyhedral, the cost coefficients $b_{t}\left(\xi_{t}\right)$ belong to $\mathbb{R}^{m_{t}}$, the right-hand sides $h_{t}\left(\xi_{t}\right)$ are in $\mathbb{R}^{n_{t}}$ and $A_{t, 0}$ and $A_{t, 1}$ are fixed $\left(n_{t}, m_{t}\right)$ - and $\left(n_{t}, m_{t-1}\right)$ matrices, respectively. We assume that the sets $X_{t}, t=2, \ldots, T$, are polyhedral cones 
and that $b_{t}(\cdot)$ and $h_{t}(\cdot)$ depend affinely linear on $\xi_{t}$. The latter condition covers the situation that some of the components of $b_{t}$ and of $h_{t}$ are random.

The challenge of multistage models consists in the presence of two groups of entirely different constraints, namely, of measurability and of pointwise constraints for the decisions $x_{t}$. This fact does not lead to consequences in the two-stage situation $(T=2)$. In general, however, it is the origin of both the theoretical and computational challenges of multistage models. In the present paper, it produces the essential difference of quantitative stability estimates compared to the two-stage case.

When solving multistage models computationally, the first step consists in approximating the stochastic process $\xi=\left\{\xi_{t}\right\}_{t=1}^{T}$ by a process having finitely many scenarios that exhibit tree structure and have its root at the fixed element $\xi_{1}$ of $\mathbb{R}^{d}$ (see the survey [4] for further information). In this way, both the random vectors $\xi^{t}$ and the $\sigma$-fields $\mathcal{F}_{t}$ are approximated at each $t$. This process finally leads to linear programming models that are very large scale in most cases and may be solved by decomposition methods that exploit specific structures of the model (see [31] for additional background). In order to reduce the model dimension, it might be desirable to reduce the originally designed tree. The approaches to scenario reduction in $[5,11]$ and to scenario tree generation in $[21,14]$ and $[10]$ make use of probability metrics, i.e., of metric distances on spaces of probability measures, where the metrics are selected such that the optimal values of original and approximate stochastic program are close if the distance of the original probability distribution $P=\mathcal{L}(\xi)$ of $\xi$ and its approximation $Q$ is small.

Such quantitative stability results are well developed for two-stage models (cf. the survey [28]). It turned out that distances of probability measures are relevant which are given by certain Monge-Kantorovich mass transportation problems. Such problems are of the form

$$
\inf \left\{\int_{\Xi \times \Xi} c(\xi, \tilde{\xi}) \eta(d \xi, d \tilde{\xi}): \eta \in \mathcal{P}(\Xi \times \Xi), \pi_{1} \eta=P, \pi_{2} \eta=Q\right\}
$$

where $\Xi$ is a closed subset of some Euclidean space, $\pi_{1}$ and $\pi_{2}$ denote the projections onto the first and second components, respectively, $c$ is a nonnegative, symmetric and continuous cost function and $P$ and $Q$ belong to a set $\mathcal{P}_{c}(\Xi)$ of probability measures on $\Xi$, where all integrals are finite. Two types of cost functions have been used in stability analysis of stochastic programs [5, 29], namely,

$$
c(\xi, \tilde{\xi}):=\|\xi-\tilde{\xi}\|^{r} \quad(\xi, \tilde{\xi} \in \Xi)
$$

and

$$
c(\xi, \tilde{\xi}):=\max \left\{1,\left\|\xi-\xi_{0}\right\|^{r-1},\left\|\tilde{\xi}-\xi_{0}\right\|^{r-1}\right\}\|\xi-\tilde{\xi}\| \quad(\xi, \tilde{\xi} \in \Xi)
$$

for some $r \geq 1$ and $\xi_{0} \in \Xi$. In both cases, the set $\mathcal{P}_{c}(\Xi)$ may be chosen as the set $\mathcal{P}_{r}(\Xi)$ of all probability measures on $\Xi$ having absolute moments of order $r$. The cost (3) leads to $L_{r}$-minimal metrics $\ell_{r}$ [25], which are defined by

$$
\ell_{r}(P, Q):=\inf \left\{\int_{\Xi \times \Xi}\|\xi-\tilde{\xi}\|^{r} \eta(d \xi, d \tilde{\xi}) \mid \eta \in \mathcal{P}(\Xi \times \Xi), \pi_{1} \eta=P, \pi_{2} \eta=Q\right\}^{\frac{1}{r}}
$$

and sometimes also called Wasserstein metrics of order $r$ [9]. The mass transportation problem (2) with cost (4) defines the Monge-Kantorovich functionals $\hat{\mu}_{r}[22,24]$. A 
variant of the functional $\hat{\mu}_{r}$ appears if, in its definition by (2), the conditions $\eta \in$ $\mathcal{P}(\Xi \times \Xi), \pi_{1} \eta=P, \pi_{2} \eta=Q$ are replaced by $\eta$ being a finite measure on $\Xi \times \Xi$ such that $\pi_{1} \eta-\pi_{2} \eta=P-Q$. The corresponding functionals $\stackrel{\circ}{\mu}_{r}$ are smaller than $\hat{\mu}_{r}$ and turn out to be metrics on $\mathcal{P}_{r}(\Xi)$. They are called Fortet-Mourier metrics of order $r$ $[8,22]$. The convergence of sequences of probability measures with respect to both metrics $\ell_{r}$ and $\stackrel{\circ}{\mu}_{r}$ is equivalent to their weak convergence and the convergence of their $r$-th order absolute moments. For $r=1$ we have the identity $\stackrel{\circ}{\mu}_{1}=\hat{\mu}_{1}=\ell_{1}$ and the corresponding metric is also called Kantorovich distance. Two-stage models are known to behave stable (even) with respect to Fortet-Mourier metrics [23].

Much less is known, however, for the multistage case. The present paper may be regarded as an extension of the quantitative analysis in [7], which considers a less general probabilistic setup and assumes implicitly that the filtrations of the original and approximate stochastic processes coincide. The paper [19] and the recent work [20] provide (qualitative) convergence results of approximations and [16, 32] deal with empirical estimates in multistage models. In the recent paper [34] the role of probability metrics for studying stability of multistage models is questioned critically. An example is given showing that closeness of original and approximate probability distributions in terms of some probability metric is not sufficient for the infima to be close in general. The recent thesis [1] focuses precisely on the question of information in stochastic programs. The conclusions of this work do not adress stability, but only discretization of multistage stochastic programs. They illuminate the role which should be played by $\sigma$-field distances in order to obtain a consistent discretization of such programs.

The main result of the present paper (Theorem 2.1) provides stability of infima of the multistage model (1) with respect to a sum of the $L_{r}$-norm and of a distance of the information structures, i.e., the filtrations of $\sigma$-fields, of the original and approximate stochastic (input) processes. Hence, it enlightens the corresponding arguments in [34]. Several comments are given on the stability result, its assumptions, the filtration distance and on the canonical choice of the underlying probability space if the original and approximate (input) probability distributions are given in practical models. Furthermore, we provide an illustrative example which shows that the filtration distance is indispensable for stability (Example 2.6). Finally, some consequences for designing scenario reduction schemes in multistage models are sketched.

\section{$2 \quad$ Stability of multistage models}

Under weak hypotheses, the program (1) can be equivalently reformulated as a minimization problem for the deterministic first stage decision $x_{1}$ (see [31, Chapter 1] or [6] for example). It is of the form

$$
\min \left\{\mathbb{E}\left[f\left(x_{1}, \xi\right)\right]=\int_{\Xi} f\left(x_{1}, \xi\right) P(d \xi): x_{1} \in X_{1}\right\}
$$

where $\Xi$ is a closed subset of $\mathbb{R}^{T d}$ containing the support of the probability distribution $P$ of $\xi$, and $f$ is an integrand on $\mathbb{R}^{m_{1}} \times \Xi$ given by the dynamic programming recursion

$$
f\left(x_{1}, \xi\right):=\Phi_{1}\left(x_{1}, \xi^{1}\right)=\left\langle b_{1}\left(\xi_{1}\right), x_{1}\right\rangle+\Phi_{2}\left(x_{1}, \xi^{2}\right),
$$




$$
\begin{aligned}
\Phi_{t}\left(x_{1}, \ldots, x_{t-1}, \xi^{t}\right):= & \inf \left\{\left\langle b_{t}\left(\xi_{t}\right), x_{t}\right\rangle+\mathbb{E}\left[\Phi_{t+1}\left(x_{1}, \ldots, x_{t}, \xi^{t+1}\right) \mid \mathcal{F}_{t}\right]: x_{t} \in X_{t},\right. \\
\left.x_{t} \text { is } \mathcal{F}_{t}-\text { measurable, } A_{t, 0} x_{t}+A_{t, 1} x_{t-1}=h_{t}\left(\xi_{t}\right)\right\} & (t=2, \ldots, T) \\
\Phi_{T+1}\left(x_{1}, \ldots, x_{T}, \xi^{T+1}\right):= & 0 .
\end{aligned}
$$

Using the representation (7) of the integrand $f$ for $T=2$ quantitative stability results are proved in $[23,28]$ with respect to Fortet-Mourier metrics of probability distributions and earlier in [29] with respect to $L_{r}$-minimal metrics. For $T>2$, however, the integrand $f$ depends on conditional expectations with respect to the $\sigma$-fields $\mathcal{F}_{t}$ and, hence, on the underlying probability measure $\mathbb{P}$ in a nonlinear way. Consequently, the methodology for studying quantitative stability properties of stochastic programs of the form (6) developed in $[23,28]$ does not apply to multistage models in general.

An alternative for studying stability of multistage models consists in considering them as optimization problems in functional spaces (see also [18],[26]), where the Banach spaces $L_{r^{\prime}}\left(\Omega, \mathcal{F}, \mathbb{P} ; \mathbb{R}^{m}\right)$ with $m=\sum_{t=1}^{T} m_{t}$ and endowed with the norm

$$
\|x\|_{r^{\prime}}:=\left(\sum_{t=1}^{T} \mathbb{E}\left[\left\|x_{t}\right\|^{r^{\prime}}\right]\right)^{\frac{1}{r^{\prime}}} \quad \text { for } r^{\prime} \in\{1,2\} \text { and }\|x\|_{\infty}:=\max _{t=1, \ldots, T} \operatorname{ess} \sup \left\|x_{t}\right\|
$$

are appropriate, where the stochastic input process $\xi$ belongs to $L_{r}\left(\Omega, \mathcal{F}, \mathbb{P} ; \mathbb{R}^{s}\right)$ with $s:=T d$, and $r$ and $r^{\prime}$ are defined by

$r=\left\{\begin{array}{ll}1 & \text { if either } h_{t} \text { or } b_{t} \text { is random, } \\ 2 & \text { if } h_{t} \text { and } b_{t} \text { are random, }\end{array} r^{\prime}:=\left\{\begin{array}{cl}\infty & , \text { only costs are random } \\ 1, & \text { only right-hand sides are random } \\ 2 & , \text { otherwise }\end{array}\right.\right.$

Let us introduce some notation. By $F: L_{r}\left(\Omega, \mathcal{F}, \mathbb{P} ; \mathbb{R}^{s}\right) \times L_{r^{\prime}}\left(\Omega, \mathcal{F}, \mathbb{P} ; \mathbb{R}^{m}\right) \rightarrow \mathbb{R}$ we denote the objective function, i.e., $F(\xi, x):=\mathbb{E}\left[\sum_{t=1}^{T}\left\langle b_{t}\left(\xi_{t}\right), x_{t}\right\rangle\right]$, by $\mathcal{X}(\xi)$ the subset of feasible elements and by $v(\xi)$ the optimal value of (1) with input $\xi$.

For our analysis, we impose the following conditions:

(A1) $A_{t, 0} X_{t}=\mathbb{R}^{n_{t}}$ for $t=2, \ldots, T$ (complete fixed recourse).

(A2) The optimal value $v(\xi)$ of (1) is finite and the objective function $F$ is levelbounded locally uniformly at $\xi$, i.e., for some $\alpha>0$ there exists a $\delta>0$ and a bounded subset $B$ of $L_{r^{\prime}}\left(\Omega, \mathcal{F}, \mathbb{P} ; \mathbb{R}^{m}\right)$ such that the level set

$$
l_{\alpha}(F(\tilde{\xi}, \cdot)):=\{\tilde{x} \in \mathcal{X}(\tilde{\xi}): F(\tilde{\xi}, \tilde{x}) \leq v(\xi)+\alpha\}
$$

is nonempty and contained in $B$ for all $\tilde{\xi} \in L_{r}\left(\Omega, \mathcal{F}, \mathbb{P} ; \mathbb{R}^{s}\right)$ with $\|\tilde{\xi}-\xi\|_{r} \leq \delta$.

(A3) $\xi \in L_{r}\left(\Omega, \mathcal{F}, \mathbb{P} ; \mathbb{R}^{s}\right)$.

Now, we are ready to state our main stability result for multistage stochastic programs.

Theorem 2.1 Let (A1), (A2) and (A3) be satisfied and $X_{1}$ be bounded.

Then there exists positive constants $L, \alpha$ and $\delta$ such that the estimate

$$
|v(\xi)-v(\tilde{\xi})| \leq L\left(\|\xi-\tilde{\xi}\|_{r}+\sum_{t=2}^{T-1} D_{t}\left(\mathcal{F}_{t}, \tilde{\mathcal{F}}_{t}\right)\right)
$$


with

$$
D_{t}\left(\mathcal{F}_{t}, \tilde{\mathcal{F}}_{t}\right):=\max \left\{\sup _{x_{t} \in l_{\alpha, t}(F(\xi, \cdot))}\left\|x_{t}-\mathbb{E}\left[x_{t} \mid \tilde{\mathcal{F}}_{t}\right]\right\|_{r^{\prime}}, \sup _{\tilde{x}_{t} \in l_{\alpha, t}(F(\tilde{\xi}, \cdot))}\left\|\tilde{x}_{t}-\mathbb{E}\left[\tilde{x}_{t} \mid \mathcal{F}_{t}\right]\right\|_{r^{\prime}}\right\}
$$

holds for all random elements $\tilde{\xi} \in L_{r}\left(\Omega, \mathcal{F}, \mathbb{P} ; \mathbb{R}^{s}\right)$ with $\|\tilde{\xi}-\xi\|_{r} \leq \delta$ and such that its optimal value $v(\tilde{\xi})$ is finite. Here, $\mathcal{F}_{t}$ and $\tilde{\mathcal{F}}_{t}, t=1, \ldots, T$, denote the filtrations of $\xi$ and $\tilde{\xi}$, respectively, and $l_{\alpha, t}(F(\xi, \cdot))$ and $l_{\alpha, t}(F(\tilde{\xi}, \cdot))$ are the sets of $t$-th components of elements of the corresponding level sets.

Proof: Let $M_{t}$ denote the set-valued mappings $u \mapsto\left\{x \in \mathbb{R}^{m_{t}} \mid A_{t, 0} x=u, x \in X_{t}\right\}$ from $\mathbb{R}^{n_{t}}$ to $\mathbb{R}^{m_{t}}$ for $t=2, \ldots, T$. The mappings have polyhedral graph and (A1) implies that their values are nonempty. Hence, the set-valued mappings $M_{t}$ are Lipschitz continuous with respect to the Hausdorff distance [27, Example 9.35]. Hence, there exist positive constants $l_{t}$ such that we have

$$
\sup _{x \in M_{t}(\bar{u})} d\left(x, M_{t}(\tilde{u})\right) \leq l_{t}\|\bar{u}-\tilde{u}\|
$$

for all $\bar{u}, \tilde{u} \in \mathbb{R}^{n_{t}}$, where $d(x, A)$ denotes the distance of $x$ to a nonempty set $A$ in $\mathbb{R}^{m_{t}}$.

Now, let $\alpha>0$ and $\delta>0$ be selected as in (A2). Let $\varepsilon \in(0, \alpha], \tilde{\xi} \in L_{r}\left(\Omega, \mathcal{F}, \mathbb{P} ; \mathbb{R}^{s}\right)$ be such that $\|\tilde{\xi}-\xi\|_{r}<\delta$ and $v(\tilde{\xi}) \in \mathbb{R}$, and let $\bar{x} \in l_{\varepsilon}(F(\xi, \cdot))$. By $\tilde{\mathcal{F}}_{t}$ we denote the $\sigma$-field generated by $\tilde{\xi}^{t}:=\left(\tilde{\xi}_{1}, \ldots, \tilde{\xi}_{t}\right)$ for $t=1, \ldots, T$. Now, we show recursively the existence of constants $L_{t}>0$ and of elements $\tilde{x}_{t}$ belonging to the appropriate spaces $L_{r^{\prime}}\left(\Omega, \tilde{\mathcal{F}}_{t}, \mathbb{P} ; \mathbb{R}^{m_{t}}\right)$ for each $t=1, \ldots, T$ such that $\tilde{x}_{t} \in X_{t}, t=1, \ldots, T$, $A_{t, 0} \tilde{x}_{t}+A_{t, 1} \tilde{x}_{t-1}=h_{t}\left(\tilde{\xi}_{t}\right), t=2, \ldots, T$, and that

$$
\left\|\mathbb{E}\left[\bar{x}_{t} \mid \tilde{\mathcal{F}}_{t}\right]-\tilde{x}_{t}\right\|
$$

can be estimated recursively with respect to $t$.

Let $t=1$, we set $\tilde{x}_{1}:=\bar{x}_{1}$ and $L_{1}:=1$. For $t>1$, we assume that $L_{t-1}$ and $\tilde{x}_{t-1}$ have already been constructed, set $\bar{u}_{t}:=h_{t}\left(\xi_{t}\right)-A_{t, 1} \bar{x}_{t-1}, \tilde{u}_{t}:=h_{t}\left(\tilde{\xi}_{t}\right)-A_{t, 1} \tilde{x}_{t-1}$ and consider the following set-valued mappings from $\Omega$ to $\mathbb{R}^{m_{t}}$ given by

$$
\omega \rightarrow M_{t}\left(\tilde{u}_{t}(\omega)\right) \quad \text { and } \quad \omega \rightarrow \arg \min _{x \in M_{t}\left(\tilde{u}_{t}(\omega)\right)}\left\|\mathbb{E}\left[\bar{x}_{t} \mid \tilde{\mathcal{F}}_{t}\right](\omega)-x\right\|
$$

Both are measurable with respect to the $\sigma$-field $\tilde{\mathcal{F}}_{t}$ due to the measurability of $\tilde{x}_{t-1}$ with respect to $\tilde{\mathcal{F}}_{t-1}$ and well known measurability results for set-valued mappings (e.g., [27, Theorem 14.36]). Hence, by appealing to [27, Theorem 14.37] there exists a $\tilde{\mathcal{F}}_{t^{-}}$ measurable selection $\tilde{x}_{t}$ of the second mapping. Since $\mathbb{E}\left[\bar{x}_{t} \mid \tilde{\mathcal{F}}_{t}\right]$ belongs to $M_{t}\left(\mathbb{E}\left[\bar{u}_{t} \mid \tilde{\mathcal{F}}_{t}\right]\right)$, (10) provides the estimate

$$
\begin{aligned}
\left\|\mathbb{E}\left[\bar{x}_{t} \mid \tilde{\mathcal{F}}_{t}\right]-\tilde{x}_{t}\right\| \leq & l_{t}\left\|\mathbb{E}\left[\bar{u}_{t} \mid \tilde{\mathcal{F}}_{t}\right]-\tilde{u}_{t}\right\| \\
\leq & l_{t}\left(\left\|\mathbb{E}\left[h_{t}\left(\xi_{t}\right) \mid \tilde{\mathcal{F}}_{t}\right]-h_{t}\left(\tilde{\xi}_{t}\right)\right\|+\left\|A_{t, 1}\right\|\left\|\mathbb{E}\left[\bar{x}_{t-1} \mid \tilde{\mathcal{F}}_{t}\right]-\tilde{x}_{t-1}\right\|\right) \\
\leq & l_{t}\left(K_{t}\left\|\mathbb{E}\left[\xi_{t} \mid \tilde{\mathcal{F}}_{t}\right]-\tilde{\xi}_{t}\right\|+\left\|A_{t, 1}\right\|\left\|\mathbb{E}\left[\bar{x}_{t-1} \mid \tilde{\mathcal{F}}_{t}\right]-\tilde{x}_{t-1}\right\|\right) \\
\leq & l_{t} \bar{K}_{t}\left(\left\|\mathbb{E}\left[\xi_{t}-\tilde{\xi}_{t} \mid \tilde{\mathcal{F}}_{t}\right]\right\|+\left\|\mathbb{E}\left[\bar{x}_{t-1}-\mathbb{E}\left[\bar{x}_{t-1} \mid \tilde{\mathcal{F}}_{t-1}\right] \mid \tilde{\mathcal{F}}_{t}\right]\right\|\right. \\
& \left.+\left\|\mathbb{E}\left[\bar{x}_{t-1} \mid \tilde{\mathcal{F}}_{t-1}\right]-\tilde{x}_{t-1}\right\|\right),
\end{aligned}
$$


where $K_{t}$ and $\bar{K}_{t}$ are certain constants. Using Jensen's inequality in the first two summands of the latter estimate we obtain recursively

$$
\left\|\mathbb{E}\left[\bar{x}_{t} \mid \tilde{\mathcal{F}}_{t}\right]-\tilde{x}_{t}\right\| \leq L_{t}\left(\sum_{\tau=2}^{t} \mathbb{E}\left[\left\|\xi_{\tau}-\tilde{\xi}_{\tau}\right\| \mid \tilde{\mathcal{F}}_{\tau}\right]+\sum_{\tau=2}^{t-1} \mathbb{E}\left[\left\|\bar{x}_{\tau}-\mathbb{E}\left[\bar{x}_{\tau} \mid \tilde{\mathcal{F}}_{\tau}\right]\right\| \mid \tilde{\mathcal{F}}_{\tau+1}\right]\right)
$$

with $L_{t}:=l_{t} \bar{K}_{t}$ for $t=2, \ldots, T$, where the first sum on the right-hand side of (11) disappears if only costs are random. Since $\left\|\tilde{x}_{t}\right\|$ may be estimated by $\left\|\tilde{x}_{t}-\mathbb{E}\left[\bar{x}_{t} \mid \tilde{\mathcal{F}}_{t}\right]\right\|+$ $\left\|\mathbb{E}\left[\bar{x}_{t} \mid \tilde{\mathcal{F}}_{t}\right]\right\|$, we conclude from (11) that $\tilde{x}_{t}$ belongs to $L_{r^{\prime}}\left(\Omega, \tilde{\mathcal{F}}_{t}, \mathbb{P} ; \mathbb{R}^{m_{t}}\right)$ in all possible cases. Inserting $\bar{x}$ and $\tilde{x}$ into the objective function we obtain

$$
v(\tilde{\xi})-v(\xi) \leq F(\tilde{\xi}, \tilde{x})-F(\xi, \bar{x})+\varepsilon .
$$

In case of only right-hand sides being random we continue (12) using (11) and obtain

$$
\begin{aligned}
v(\tilde{\xi})-v(\xi) & \leq \sum_{t=1}^{T} \mathbb{E}\left[\left\langle b_{t}, \mathbb{E}\left[\tilde{x}_{t}-\bar{x}_{t} \mid \tilde{\mathcal{F}}_{t}\right]\right\rangle\right]+\varepsilon \leq \sum_{t=1}^{T}\left\|b_{t}\right\| \mathbb{E}\left[\left\|\tilde{x}_{t}-\mathbb{E}\left[\bar{x}_{t} \mid \tilde{\mathcal{F}}_{t}\right]\right\|\right]+\varepsilon \\
& \leq \hat{L} \sum_{t=1}^{T} \mathbb{E}\left[\sum_{\tau=2}^{t} \mathbb{E}\left[\left\|\xi_{\tau}-\tilde{\xi}_{\tau}\right\| \mid \tilde{\mathcal{F}}_{\tau}\right]+\sum_{\tau=2}^{t-1} \mathbb{E}\left[\left\|\bar{x}_{\tau}-\mathbb{E}\left[\bar{x}_{\tau} \mid \tilde{\mathcal{F}}_{\tau}\right]\right\| \mid \tilde{\mathcal{F}}_{\tau+1}\right]\right]+\varepsilon \\
& \leq \hat{L} T \mathbb{E}\left[\sum_{t=1}^{T}\left\|\xi_{t}-\tilde{\xi}_{t}\right\|+\sum_{\tau=2}^{T-1}\left\|\bar{x}_{\tau}-\mathbb{E}\left[\bar{x}_{\tau} \mid \tilde{\mathcal{F}}_{\tau}\right]\right\|\right]+\varepsilon \\
& \leq \hat{L} T\left(\mathbb{E}[\|\xi-\tilde{\xi}\|]+\mathbb{E}\left[\sum_{\tau=2}^{T-1}\left\|\bar{x}_{\tau}-\mathbb{E}\left[\bar{x}_{\tau} \mid \tilde{\mathcal{F}}_{\tau}\right]\right\|\right]\right)+\varepsilon
\end{aligned}
$$

where $\hat{L}:=\max _{t=1, \ldots, T} L_{t}\left\|b_{t}\right\|$. Analogously, for the cases $r=2$ and only costs being random we obtain the estimate

$$
\begin{aligned}
v(\tilde{\xi})-v(\xi) & \leq F(\tilde{\xi}, \tilde{x})-F(\tilde{\xi}, \bar{x})+F(\tilde{\xi}, \bar{x})-F(\xi, \bar{x})+\varepsilon \\
& \leq \mathbb{E}\left[\sum_{t=1}^{T}\left\langle b_{t}\left(\tilde{\xi}_{t}\right), \mathbb{E}\left[\tilde{x}_{t}-\bar{x}_{t} \mid \tilde{\mathcal{F}}_{t}\right]\right\rangle\right]+\mathbb{E}\left[\sum_{t=1}^{T}\left\langle b_{t}\left(\tilde{\xi}_{t}\right)-b_{t}\left(\xi_{t}\right), \bar{x}_{t}\right\rangle\right]+\varepsilon \\
& \leq \hat{K} \mathbb{E}\left[\sum_{t=1}^{T} \max \left\{1,\left\|\tilde{\xi}_{t}\right\|\right\}\left\|\tilde{x}_{t}-\mathbb{E}\left[\bar{x}_{t} \mid \tilde{\mathcal{F}}_{t}\right]\right\|+\sum_{t=1}^{T}\left\|\tilde{\xi}_{t}-\xi_{t}\right\|\left\|\bar{x}_{t}\right\|\right]+\varepsilon
\end{aligned}
$$

with some positive constant $\hat{K}$. In case of only random costs, i.e., $r=1$ and $r^{\prime}=\infty$, we continue

$$
\begin{aligned}
v(\tilde{\xi})-v(\xi) & \leq \hat{K} \mathbb{E}\left[\sum_{t=1}^{T} \max \left\{1,\left\|\tilde{\xi}_{t}\right\|\right\}\left\|\tilde{x}_{t}-\mathbb{E}\left[\bar{x}_{t} \mid \tilde{\mathcal{F}}_{t}\right]\right\|\right]+\hat{K} \mathbb{E}[\|\tilde{\xi}-\xi\|]\|\bar{x}\|_{\infty}+\varepsilon \\
& \leq \hat{K} \sum_{t=1}^{T} \mathbb{E}\left[\max \left\{1,\left\|\tilde{\xi}_{t}\right\|\right\}\right]\left\|\tilde{x}_{t}-\mathbb{E}\left[\bar{x}_{t} \mid \tilde{\mathcal{F}}_{t}\right]\right\|_{\infty}+K \mathbb{E}[\|\tilde{\xi}-\xi\|]+\varepsilon,
\end{aligned}
$$

where (A2) was used to bound $\|\bar{x}\|_{\infty}$ leading to a constant $K>0$. Next we make use of the fact that $\tilde{\xi}$ varies in a bounded set in $L_{1}$ and of the estimate (11). This leads to

$$
v(\tilde{\xi})-v(\xi) \leq L\left(\|\tilde{\xi}-\xi\|_{1}+\sum_{\tau=2}^{T-1}\left\|\bar{x}_{\tau}-\mathbb{E}\left[\bar{x}_{\tau} \mid \tilde{\mathcal{F}}_{\tau}\right]\right\|_{\infty}\right)+\varepsilon
$$


with some constant $L>0$. Finally, it remains to consider the case $r=r^{\prime}=2$. Starting from (13) we use the Cauchy-Schwarz inequality and obtain

$$
\begin{aligned}
v(\tilde{\xi})-v(\xi) \leq \hat{K}\left[\left(\sum_{t=1}^{T} \mathbb{E}\left[\max \left\{1,\left\|\tilde{\xi}_{t}\right\|^{2}\right\}\right]\right)^{\frac{1}{2}}\left(\sum_{t=1}^{T} \mathbb{E}\left[\left\|\tilde{x}_{t}-\mathbb{E}\left[\bar{x}_{t} \mid \tilde{\mathcal{F}}_{t}\right]\right\|^{2}\right]\right)^{\frac{1}{2}}\right. \\
\left.+\|\tilde{\xi}-\xi\|_{2}\|\bar{x}\|_{2}\right]+\varepsilon \\
\leq L\left(\|\tilde{\xi}-\xi\|_{2}+\sum_{t=2}^{T-1}\left\|\bar{x}_{t}-\mathbb{E}\left[\bar{x}_{t} \mid \tilde{\mathcal{F}}_{t}\right]\right\|_{2}\right)+\varepsilon
\end{aligned}
$$

with some constant $L>0$ due to (11), (A2) and the fact that $\tilde{\xi}$ varies in some bounded set in $L_{2}$. Altogether, we obtain for all cases

$$
v(\tilde{\xi})-v(\xi) \leq L\left(\|\xi-\tilde{\xi}\|_{r}+\sum_{t=2}^{T-1} \sup _{\bar{x} \in l_{\alpha, t}(F(\xi,))}\left\|\bar{x}_{t}-\mathbb{E}\left[\bar{x}_{t} \mid \tilde{\mathcal{F}}_{t}\right]\right\|_{r^{\prime}}\right)+\varepsilon
$$

with some constant $L>0$. Since $\varepsilon>0$ was chosen arbitrarily, we conclude

$$
v(\tilde{\xi})-v(\xi) \leq L\left(\|\xi-\tilde{\xi}\|_{r}+\sum_{t=2}^{T-1} \sup _{\bar{x} \in l_{\alpha, t}(F(\xi, \cdot))}\left\|\bar{x}_{t}-\mathbb{E}\left[\bar{x}_{t} \mid \tilde{\mathcal{F}}_{t}\right]\right\|_{r^{\prime}}\right) .
$$

Changing the role of $\xi$ and $\tilde{\xi}$ leads to an estimate of the form

$$
v(\xi)-v(\tilde{\xi}) \leq L\left(\|\xi-\tilde{\xi}\|_{r}+\sum_{t=2}^{T-1} \sup _{\tilde{x} \in l_{\alpha, t}(F(\tilde{\xi},))}\left\|\tilde{x}_{t}-\mathbb{E}\left[\tilde{x}_{t} \mid \mathcal{F}_{t}\right]\right\|_{r^{\prime}}\right) .
$$

Finally, it remains to note that the second summands in the estimates (14) and (15) are bounded by

$$
\sum_{t=2}^{T-1} D_{t}\left(\mathcal{F}_{t}, \tilde{\mathcal{F}}_{t}\right)
$$

Thus, the proof is complete.

Remark 2.2 Our assumption on the locally uniform level-boundedness of the objective function $F$ is quite standard in perturbation results for optimization problems (see, e.g., [27, Theorem 1.17]). The finiteness condition for the optimal values is needed because it cannot be concluded from the level-boundedness of $F$ for all relevant pairs $\left(r, r^{\prime}\right)$. In case $r=r^{\prime}=2$, the existence of solutions of (1) (and, thus, the finiteness of $v(\xi)$ ) is a simple consequence of the weak compactness of $l_{\alpha}(F(\xi, \cdot))$ in $L_{2}\left(\Omega, \mathcal{F}, \mathbb{P} ; \mathbb{R}^{m}\right)$ and the linearity of the objective.

If solutions of (1) and its approximation exist, an inspection of the proof shows that $\bar{x}$ and $\tilde{x}$ may be chosen as such solutions and the distance $D_{t}$ attains the form

$$
D_{t}\left(\mathcal{F}_{t}, \tilde{\mathcal{F}}_{t}\right)=\max \left\{\left\|x_{t}-\mathbb{E}\left[x_{t} \mid \tilde{\mathcal{F}}_{t}\right]\right\|_{r^{\prime}},\left\|\tilde{x}_{t}-\mathbb{E}\left[\tilde{x}_{t} \mid \mathcal{F}_{t}\right]\right\|_{r^{\prime}}\right\} .
$$

Hence, the distance of the filtrations $\left\{\mathcal{F}_{t}\right\}_{t=1}^{T}$ and $\left\{\tilde{\mathcal{F}}_{t}\right\}_{t=1}^{T}$ is evaluated on the original and the perturbed solution, respectively. An equivalent formulation of (16) is

$$
D_{t}\left(\mathcal{F}_{t}, \tilde{\mathcal{F}}_{t}\right)=\max \left\{\left\|x_{t}-\mathbb{E}\left[x_{t} \mid \tilde{\xi}_{1}, \ldots, \tilde{\xi}_{t}\right]\right\|_{r^{\prime}},\left\|\tilde{x}_{t}-\mathbb{E}\left[\tilde{x}_{t} \mid \xi_{1}, \ldots, \xi_{t}\right]\right\|_{r^{\prime}}\right\} .
$$


Remark 2.3 In practical situations, the available knowledge on the stochastic input consists in (partial or complete) information on its probability distribution. Which probability space should be selected? A natural answer certainly is: A probability space where the $L_{r}$-distance is minimal and an optimal coupling exists. To be more precise let $P$ and $Q$ be probability distributions in $\mathcal{P}_{r}(\Xi)$. Then there exists an optimal solution $\eta^{*} \in \mathcal{P}(\Xi \times \Xi)$ of the mass transportation problem (5) [22, Theorem 8.1.1], i.e.,

$$
\ell_{r}^{r}(P, Q)=\int_{\Xi \times \Xi}\|\xi-\tilde{\xi}\|^{r} \eta^{*}(d \xi, d \tilde{\xi}),
$$

where $\pi_{1} \eta^{*}=P$ and $\pi_{2} \eta^{*}=Q$. Furthermore, there exists a probability space $\left(\Omega^{\prime}, \mathcal{F}^{\prime}, \mathbb{P}^{\prime}\right)$ and an optimal coupling, i.e., a pair $\left(\xi^{\prime}(\cdot), \tilde{\xi}^{\prime}(\cdot)\right)$ of $\Xi$-valued random elements defined on it, such that the probability distribution of $\left(\xi^{\prime}(\cdot), \tilde{\xi}^{\prime}(\cdot)\right)$ is just $\eta^{*}$ [22, Theorem 2.5.1]. In particular, we have

$$
\ell_{r}(P, Q)=\left\|\xi^{\prime}(\cdot)-\tilde{\xi}^{\prime}(\cdot)\right\|_{r}
$$

and the filtration distance is evaluated on the filtrations $\left\{\mathcal{F}_{t}^{\prime}\right\}_{t=1}^{T}$ and $\left\{\tilde{\mathcal{F}}_{t}^{\prime}\right\}_{t=1}^{T}$ generated by $\xi^{\prime}$ and $\tilde{\xi}^{\prime}$, respectively.

Although there exist general characterization results of optimal couplings in [24, Section 3.3], the specific result for the $L_{2}$-situation is particularly powerful. Namely, a pair $\left(\xi^{\prime}, \tilde{\xi}^{\prime}\right)$ of $\mathbb{R}^{k}$-valued random vectors is an optimal $L_{2}$-coupling if and only if there exists a lower semicontinuous convex function $f: \mathbb{R}^{k} \rightarrow \overline{\mathbb{R}}$ such that $\tilde{\xi}^{\prime} \in \partial f\left(\xi^{\prime}\right)$, $\mathbb{P}$-a.s., holds with $\partial f(\cdot)$ denoting the subdifferential of $f$ [24, Theorem 3.2.9].

Remark 2.4 (Stability of first-stage solutions) Using the same technique as for proving [28, Theorem 9], the continuity property of infima in Theorem 2.1 can be supplemented by a quantitative stability property of the solution set $S(\xi)$ of (6), i.e., of the set of first stage solutions. Namely, there exists a constant $\hat{L}>0$ such that

$$
\sup _{x \in S(\tilde{\xi})} d(x, S(\xi)) \leq \Psi_{\xi}^{-1}\left(\hat{L}\left(\|\xi-\tilde{\xi}\|_{r}+\sum_{t=2}^{T-1} D_{t}\left(\mathcal{F}_{t}, \tilde{\mathcal{F}}_{t}\right)\right)\right)
$$

where $\Psi_{\xi}(\tau):=\inf \left\{\mathbb{E}\left[f\left(x_{1}, \xi\right)\right]-v(\xi): d\left(x_{1}, S(\xi)\right) \geq \tau, x_{1} \in X_{1}\right\}$ with $\Psi_{\xi}^{-1}(\alpha):=$ $\sup \left\{\tau \in \mathbb{R}_{+}: \Psi_{\xi}(\tau) \leq \alpha\right\}\left(\alpha \in \mathbb{R}_{+}\right)$is the growth function of the original problem (6) close to its solution set $S(\xi)$.

The boundedness condition for $X_{1}$ in Theorem 2.1 can be relaxed to the assumption that the set $S(\xi)$ is bounded. In the latter case a version of (8) is derived that contains localized optimal values. Then the estimate (8) is valid whenever its right-hand side is sufficiently small.

Remark 2.5 (Convergence of filtrations) This remark aims at precising the link between the second summand in the right hand-side of our stability estimate (8), and previous work on convergence of information.

A distance between $\sigma$-fields was introduced in [2]. It metrizes a topology called uniform topology on the set of $\sigma$-fields. Due to the work of [30] and [17], this distance reads, for all $\mathcal{B}, \mathcal{B}^{\prime}$ sub- $\sigma$-fields of $\mathcal{F}$

$$
d_{B}\left(\mathcal{B}, \mathcal{B}^{\prime}\right):=\sup _{f \in \Phi} \mathbb{E}\left[\left\|\mathbb{E}[f \mid \mathcal{B}]-\mathbb{E}\left[f \mid \mathcal{B}^{\prime}\right]\right\|\right]
$$


with $\Phi$ the set of all $\mathcal{F}$-measurable functions $f$ such that $\forall \omega \in \Omega,\|f(\omega)\| \leq 1$. Thanks to [15], a filtration can be said to converge to another one if and only if each $\sigma$-field at each time step converges according to the distance $d_{B}$. Hence, a distance between filtrations can be introduced, based on the sum of the distances between $\sigma$-fields. The second summand in our stability result can be seen as such a distance between the filtrations generated by the two stochastic processes $\xi$ and $\tilde{\xi}$. This summand is not exactly the same as the sum of distances $d_{B}$, but it has the same sense: If the feasible set of our multistage stochastic program is bounded, the second summand in Theorem 2.1 is bounded by a sum of distances $d_{B}$.

Other distances between filtrations and $\sigma$-fields have been introduced (see, e.g., [3]) to fit with stochastic optimization problems. The thesis [1] provides a good survey and a few new results on the application of such information distances.

The following example shows that filtration distances are indispensable for the stability of multistage models.

Example 2.6 We consider a multistage stochastic program that models the optimal purchase over time under cost uncertainty. Its decisions $x_{t}$ correspond to the amounts to be purchased at each time period. The uncertain prices are $\xi_{t}, t=1, \ldots, T$, and the objective consists in minimizing the expected costs such that a prescribed amount a is achieved at the end of a given time horizon. The problem is of the form

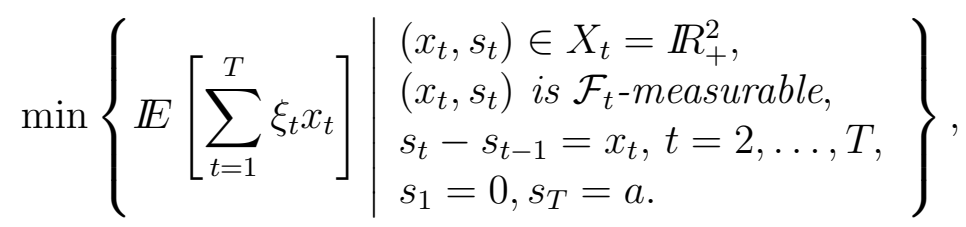

where the state variable $s_{t}$ corresponds to the amount at time $t$ and $\mathcal{F}_{t}:=\sigma\left\{\xi_{1}, \ldots, \xi_{t}\right\}$. Let $T:=3$ and $P_{\varepsilon}$ denote the probability distribution of the stochastic price process. $P_{\varepsilon}$ is given by the two scenarios $\xi_{\varepsilon}^{1}=(3,2+\varepsilon, 3)(\varepsilon \in(0,1))$ and $\xi_{\varepsilon}^{2}=(3,2,1)$ each endowed with probability $\frac{1}{2}$. Let $Q$ denote the approximation of $P$ given by the two scenarios $\tilde{\xi}^{1}=(3,2,3)$ and $\tilde{\xi}^{2}=(3,2,1)$ with the same probabilities $\frac{1}{2}$.

We assume that the scenario trees of the processes $\xi_{\varepsilon}$ and $\tilde{\xi}$ are of the form displayed in Figure 1, i.e., the filtrations of $\sigma$-fields generated by $\xi_{\varepsilon}$ and $\tilde{\xi}$ do not coincide.
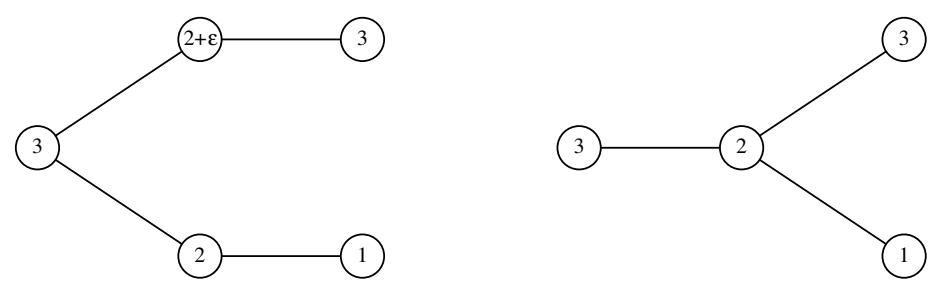

Figure 1: Scenario trees for $P_{\varepsilon}$ (left) and $Q$

We obtain

$$
v\left(\xi_{\varepsilon}\right)=\frac{3+\varepsilon}{2} a \quad \text { and } \quad v(\tilde{\xi})=2 a, \text { but } \quad \ell_{1}\left(P_{\varepsilon}, Q\right)=\frac{\varepsilon}{2} .
$$

Hence, the multistage stochastic purchasing model is not stable with respect to the $L_{1}$ minimal distance $\ell_{1}$. However, the estimate for $|v(\xi)-v(\tilde{\xi})|$ in Theorem 2.1 is valid with $L=a$ since we obtain $D_{2}\left(\mathcal{F}_{2}, \tilde{\mathcal{F}}_{2}\right)=1$ for the filtration distance. 
Finally, let us consider the case of discrete probability measures $P$ and $Q$. Let $P$ have scenarios $\xi^{i}$ with probabilities $p_{i}>0, i=1, \ldots, N$, and $Q$ scenarios $\tilde{\xi}^{j}$ and probabilities $q_{j}>0, j=1, \ldots, M$. Clearly, $\sum_{i=1}^{N} p_{i}=1$ and $\sum_{j=1}^{M} q_{j}=1$. Then $\ell_{r}^{r}(P, Q)$ is the optimal value of a finite-dimensional linear transportation problem (e.g., [24]) and there exist optimal weights $\eta_{i j} \geq 0$ of the scenario pair $\left(\xi^{i}, \tilde{\xi}^{j}\right), i=1, \ldots, N, j=1, \ldots, M$. Hence, there exists a pair $(\xi, \tilde{\xi})$ of random vectors on the probability space $(\Omega, \mathcal{F}, \mathbb{P})$, where $\Omega=\left\{\omega_{i j}: i=1, \ldots, N, j=1, \ldots, M\right\}$ and $\mathbb{P}\left(\omega_{i j}\right)=\eta_{i j}, i=1, \ldots, N, j=$ $1, \ldots, M$. We define $\xi\left(\omega_{i j}\right)=\xi^{i}$ for every $j=1, \ldots, M$ and $\tilde{\xi}\left(\omega_{i j}\right)=\tilde{\xi}^{j}$ for every $i=1, \ldots, N$.

Now, our aim is to study the second term in the stability estimate in Theorem 2.1 , namely, the distance of filtrations. Let $\mathcal{F}_{t}$ and $\tilde{\mathcal{F}}_{t}$ denote the $\sigma$-fields generated by $\left(\xi_{1}, \ldots, \xi_{t}\right)$ and $\left(\tilde{\xi}_{1}, \ldots, \tilde{\xi}_{t}\right)$, respectively. Let $I_{t}$ and $\tilde{I}_{t}$ denote the index set of realizations of $\xi_{t}$ and $\tilde{\xi}_{t}$, respectively. Furthermore, let $\mathcal{E}_{t}$ and $\tilde{\mathcal{E}}_{t}$ denote families of nonempty elements of $\mathcal{F}_{t}$ and $\tilde{\mathcal{F}}_{t}$, respectively, that form partitions of $\Omega$ and generate the corresponding $\sigma$-fields. We set $E_{t s}:=\left\{\omega \in \Omega:\left(\xi_{1}(\omega), \ldots, \xi_{t}(\omega)\right)=\left(\xi_{1}^{s}, \ldots, \xi_{t}^{s}\right)\right\}$, $s \in I_{t}$, and $\tilde{E}_{t s}:=\left\{\omega \in \Omega:\left(\tilde{\xi}_{1}(\omega), \ldots, \tilde{\xi}_{t}(\omega)\right)=\left(\tilde{\xi}_{1}^{s}, \ldots, \tilde{\xi}_{t}^{s}\right)\right\}, s \in \tilde{I}_{t}$.

We set $r=r^{\prime}=1$ and require conditions (A1) and (A2) to hold. Since (1) is finitedimensional in this case, optimal solutions $x$ and $\tilde{x}$ exist and we obtain according to Remark 2.2 that

$$
\begin{aligned}
D_{t}\left(\mathcal{F}_{t}, \tilde{\mathcal{F}}_{t}\right)=\max \{ & \sum_{i, j} \eta_{i j}\left\|x_{t}\left(\omega_{i j}\right)-\mathbb{E}\left[x_{t} \mid \tilde{\mathcal{F}}_{t}\right]\left(\omega_{i j}\right)\right\|, \\
& \left.\sum_{i, j} \eta_{i j}\left\|\tilde{x}_{t}\left(\omega_{i j}\right)-\mathbb{E}\left[\tilde{x}_{t} \mid \mathcal{F}_{t}\right]\left(\omega_{i j}\right)\right\|\right\} \\
= & \max \left\{\sum_{s \in \tilde{I}_{t}} \sum_{\omega_{i j} \in \tilde{E}_{t s}} \eta_{i j}\left\|x_{t}\left(\omega_{i j}\right)-\frac{\sum_{\omega_{k l} \in \tilde{E}_{t s}} \eta_{k l} x_{t}\left(\omega_{k l}\right)}{\sum_{\omega_{k l} \in \tilde{E}_{t s}} \eta_{k l}}\right\|,\right. \\
& \left.\sum_{s \in I_{t}} \sum_{\omega_{i j} \in E_{t s}} \eta_{i j}\left\|\tilde{x}_{t}\left(\omega_{i j}\right)-\frac{\sum_{\omega_{k l} \in E_{t s}} \eta_{k l} \tilde{x}_{t}\left(\omega_{k l}\right)}{\sum_{\omega_{k l} \in E_{t s}} \eta_{k l}}\right\|\right\}
\end{aligned}
$$

The latter representation of $D_{t}$ has potential to be further estimated in specific cases. In particular, it simplifies considerably for the situation of scenario reduction.

Example 2.7 (scenario reduction) Let us consider the case of deleting scenario $l \in$ $\{1, \ldots, N\}$ of $\xi$ according to the methodology in [5, 11] for the distance $\ell_{1}$ and $r=r^{\prime}=$ 1. Then $\tilde{\xi}$ has the scenarios $\xi^{1}, \ldots, \xi^{l-1}, \xi^{l+1}, \ldots, \xi^{N}$ and the probabilities of $\xi^{j}$ are $q_{j}=p_{j}$ for every $j \neq j(l)$ and $q_{j(l)}=p_{j(l)}+p_{l}$, where $j(l) \in \arg \min _{j \neq l} c_{r}\left(\xi^{j}, \xi^{l}\right)$ (see [5, Theorem 2]). This corresponds to $\tilde{\xi}\left(\omega_{i j}\right)=\xi^{j}$ for every $i=1, \ldots, N, j=1, \ldots, N$, $j \neq l$, and $\tilde{\xi}\left(\omega_{i l}\right)=\xi^{j(l)}$ for $i=1, \ldots, N$. We also infer from [5, Theorem 2] that the optimal weights of the transportation problem defining $\ell_{1}(P, Q)$ are

$$
\eta_{i j}=\left\{\begin{array}{cl}
p_{l} & , i=l, j=j(l), \\
p_{j} & , i=j \neq l \\
0 & , \text { otherwise }
\end{array}\right.
$$


We set $\hat{\omega}_{j}:=\omega_{j j}$ for every $j=1, \ldots, N, j \neq l, \hat{\omega}_{l}=\omega_{l j(l)}$ and introduce the notation $E_{t s_{j}}$ and $\tilde{E}_{t s_{j}}$ for the sets in $\mathcal{E}_{t}$ and $\tilde{\mathcal{E}}_{t}$, respectively, that contain $\hat{\omega}_{j}$.

From (20) we conclude the following representations of $D_{t}$

$$
\begin{aligned}
D_{t}\left(\mathcal{F}_{t}, \tilde{\mathcal{F}}_{t}\right)= & \max \left\{\sum_{s \in \tilde{I}_{t}} \sum_{\hat{\omega}_{j} \in \tilde{E}_{t s}} p_{j}\left\|x_{t}\left(\hat{\omega}_{j}\right)-\frac{\sum_{\hat{\omega}_{k} \in \tilde{E}_{t s}} p_{k} x_{t}\left(\hat{\omega}_{k}\right)}{\sum_{\hat{\omega}_{k} \in \tilde{E}_{t s}} p_{k}}\right\|,\right. \\
& \left.\sum_{s \in I_{t}} \sum_{\hat{\omega}_{j} \in E_{t s}} p_{j}\left\|\tilde{x}_{t}\left(\hat{\omega}_{j}\right)-\frac{\sum_{\omega_{k} \in E_{t s}} p_{k} \tilde{x}_{t}\left(\hat{\omega}_{k}\right)}{\sum_{\hat{\omega}_{k} \in E_{t s}} p_{k}}\right\|\right\} \\
= & \max \left\{\sum_{s \in \tilde{I}_{t}} \frac{1}{\sum_{\hat{\omega}_{k} \in \tilde{E}_{t s}} p_{k}} \sum_{\hat{\omega}_{j} \in \tilde{E}_{t s}}\left\|\sum_{\hat{\omega}_{k} \in \tilde{E}_{t s}} p_{k} p_{j}\left[x_{t}\left(\hat{\omega}_{j}\right)-x_{t}\left(\hat{\omega}_{k}\right)\right]\right\|,\right. \\
& \left.\sum_{s \in I_{t}} \frac{1}{\sum_{\hat{\omega}_{k} \in E_{t s}} p_{k}} \sum_{\hat{\omega}_{j} \in E_{t s}}\left\|\sum_{\hat{\omega}_{k} \in E_{t s}} p_{k} p_{j}\left[\tilde{x}_{t}\left(\hat{\omega}_{j}\right)-\tilde{x}_{t}\left(\hat{\omega}_{k}\right)\right]\right\|\right\} \\
& \max \left\{\sum_{s \in \tilde{I}_{t}} \frac{1}{\sum_{\hat{\omega}_{k} \in \tilde{E}_{t s}} p_{k}} \sum_{\hat{\omega}_{j} \in \tilde{E}_{t s}}\left\|\sum_{\hat{\omega}_{k} \in \tilde{E}_{t s} \backslash E_{t s_{j}}} p_{k} p_{j}\left[x_{t}\left(\hat{\omega}_{j}\right)-x_{t}\left(\hat{\omega}_{k}\right)\right]\right\|,\right. \\
& \left.\sum_{s \in I_{t}} \frac{1}{\sum_{\hat{\omega}_{k} \in E_{t s}} p_{k}} \sum_{\hat{\omega}_{j} \in E_{t s}}\left\|\sum_{\hat{\omega}_{k} \in E_{t s} \backslash \tilde{E}_{t s_{j}}} p_{k} p_{j}\left[\tilde{x}_{t}\left(\hat{\omega}_{j}\right)-\tilde{x}_{t}\left(\hat{\omega}_{k}\right)\right]\right\|\right\},
\end{aligned}
$$

where the final equality is a consequence of the corresponding measurability properties of $x_{t}$, which imply $x_{t}\left(\hat{\omega}_{j}\right)=x_{t}\left(\hat{\omega}_{k}\right)$ if $\hat{\omega}_{k} \in E_{t s} \cap \tilde{E}_{t s_{j}}$ and $\hat{\omega}_{k} \in \tilde{E}_{t s} \cap E_{t s_{j}}$, respectively. Since $E_{t s_{j}}=\tilde{E}_{t s_{j}}$ for $j \notin\{l, j(l)\}$ and $\tilde{E}_{t s_{l}}=E_{t j(l)} \cup\left\{\hat{\omega}_{l}\right\}$, we may continue

$$
\begin{aligned}
& D_{t}\left(\mathcal{F}_{t}, \tilde{\mathcal{F}}_{t}\right)=\max \left\{\frac{1}{\sum_{\hat{\omega}_{k} \in \tilde{E}_{t s_{l}}} p_{k}} \sum_{\hat{\omega}_{j} \in \tilde{E}_{t s_{l}}}\left\|\sum_{\hat{\omega}_{k} \in \tilde{E}_{t s_{l} \backslash E_{t s_{j}}}} p_{k} p_{j}\left[x_{t}\left(\hat{\omega}_{j}\right)-x_{t}\left(\hat{\omega}_{k}\right)\right]\right\|,\right. \\
& \left.\frac{1}{\sum_{\hat{\omega}_{k} \in E_{t s_{l}}} p_{k}} \sum_{\hat{\omega}_{j} \in E_{t s_{l}}}\left\|\sum_{\hat{\omega}_{k} \in E_{t s_{l} \backslash \tilde{E}_{t s_{j}}}} p_{k} p_{j}\left[\tilde{x}_{t}\left(\hat{\omega}_{j}\right)-\tilde{x}_{t}\left(\hat{\omega}_{k}\right)\right]\right\|\right\} \\
& =\max \left\{\frac { 1 } { \sum _ { \hat { \omega } _ { k } \in \tilde { E } _ { t s _ { l } } } p _ { k } } \left\{\sum_{\hat{\omega}_{k} \in E_{t s_{j(l)}}}\left\|p_{l} p_{k}\left[x_{t}\left(\hat{\omega}_{k}\right)-x_{t}\left(\hat{\omega}_{l}\right)\right]\right\|\right.\right. \\
& \left.+\left\|\sum_{\hat{\omega}_{k} \in E_{t s_{j}(l)}} p_{k} p_{l}\left[\tilde{x}_{t}\left(\hat{\omega}_{l}\right)-\tilde{x}_{t}\left(\hat{\omega}_{k}\right)\right]\right\|\right\} \\
& \frac{1}{\sum_{\hat{\omega}_{k} \in E_{t s_{l}}} p_{k}}\left\{\sum_{\hat{\omega}_{k} \in E_{t s_{l}} \backslash\left\{\hat{\omega}_{l}\right\}}\left\|p_{l} p_{k}\left[x_{t}\left(\hat{\omega}_{k}\right)-x_{t}\left(\hat{\omega}_{l}\right)\right]\right\|\right. \\
& \left.\left.+\left\|\sum_{\hat{\omega}_{k} \in E_{t s_{l} \backslash\left\{\hat{\omega}_{l}\right\}}} p_{k} p_{l}\left[\tilde{x}_{t}\left(\hat{\omega}_{l}\right)-\tilde{x}_{t}\left(\hat{\omega}_{k}\right)\right]\right\|\right\}\right\}
\end{aligned}
$$




$$
\begin{aligned}
& \leq \max \left\{\frac{\sum_{\hat{\omega}_{k} \in E_{t s_{j(l)}}} 2 p_{l} p_{k}\left\|x_{t}\left(\hat{\omega}_{k}\right)-x_{t}\left(\hat{\omega}_{l}\right)\right\|}{p_{l}+\sum_{\hat{\omega}_{k} \in E_{t s_{j l}(l)}} p_{k}}, \frac{\sum_{\hat{\omega}_{k} \in E_{\left.t s_{l} \backslash \backslash \hat{\omega}_{l}\right\}}} 2 p_{l} p_{k}\left\|\tilde{x}_{t}\left(\hat{\omega}_{k}\right)-\tilde{x}_{t}\left(\hat{\omega}_{l}\right)\right\|}{p_{l}+\sum_{\hat{\omega}_{k} \in E_{t s_{l} \backslash\left\{\hat{\omega}_{l}\right\}}} p_{k}}\right\} \\
& \leq 2 p_{l} \max \left\{\left\|x_{t}\left(\hat{\omega}_{j(l)}\right)-x_{t}\left(\hat{\omega}_{l}\right)\right\|, \min _{\left.\hat{\omega}_{k} \in E_{t s_{l} \backslash\left\{\hat{\omega}_{l}\right\}}\left\|\tilde{x}_{t}\left(\hat{\omega}_{k}\right)-\tilde{x}_{t}\left(\hat{\omega}_{l}\right)\right\|\right\},}\right.
\end{aligned}
$$

where the convention is used that $\min _{\hat{\omega}_{k} \in E_{t s_{l}} \backslash\left\{\hat{\omega}_{l}\right\}}=0$ if $E_{t s_{l}} \backslash\left\{\hat{\omega}_{l}\right\}=\emptyset$. The final estimate makes use of the fact that all $x_{t}\left(\hat{\omega}_{k}\right)$ with $\hat{\omega}_{k} \in E_{t s_{j(l)}}$ and $\hat{\omega}_{k} \in E_{t s_{l}} \backslash\left\{\hat{\omega}_{l}\right\}$, respectively, coincide.

In the following two cases, the above estimate simplifies to

$$
D_{t}\left(\mathcal{F}_{t}, \tilde{\mathcal{F}}_{t}\right) \leq\left\{\begin{array}{cl}
0 & \text { if } \hat{\omega}_{l} \in E_{t s_{j(l)}}, \\
2 p_{l}\left\|x_{t}\left(\hat{\omega}_{j(l)}\right)-x_{t}\left(\hat{\omega}_{l}\right)\right\| & \text {, if } E_{t s_{l}}=\left\{\hat{\omega}_{l}\right\} .
\end{array}\right.
$$

As the sets $l_{0}(F(\xi, \cdot))$ and $l_{0}(F(\tilde{\xi}, \cdot))$ of solutions of the original and perturbed multistage models are bounded in $L_{r^{\prime}}$ due to (A2), there exists a constant $K>0$ such that

$$
\sum_{t=2}^{T-1} D_{t}\left(\mathcal{F}_{t}, \tilde{\mathcal{F}}_{t}\right) \leq K p_{l}
$$

Hence, if the probability $p_{l}$ of the deleted scenario is small, the filtration distance is also small. Then there is no need to modify the deletion procedure based on best approximations with respect to the metric $\ell_{1}$. A more reliable estimate for the filtration distance may be obtained by solving the stochastic program for an approximation $\hat{\xi}$ of $\xi\left(\right.$ on $\left.\left\{\hat{\omega}_{1}, \ldots, \hat{\omega}_{N}\right\}\right)$, which contains much less scenarios than $\xi$. Then an estimate for the filtration distance may be obtained by computing

$$
2 p_{l} \sum_{t=2}^{T-1} \max \left\{\left\|\hat{x}_{t}\left(\hat{\omega}_{j(l)}\right)-\hat{x}_{t}\left(\hat{\omega}_{l}\right)\right\|, \min _{\hat{\omega}_{k} \in E_{t s_{l}} \backslash\left\{\hat{\omega}_{l}\right\}}\left\|\hat{x}_{t}\left(\hat{\omega}_{k}\right)-\hat{x}_{t}\left(\hat{\omega}_{l}\right)\right\|\right\},
$$

where $\hat{x} \in l_{0}(F(\hat{\xi}, \cdot))$ is the corresponding solution. Altogether, some scenario deletion suggested by the strategy in [5] and [11] can be either rejected or accepted according to its effect on the filtration distance.

\section{Conclusions}

While quantitative stability results for two-stage stochastic programs have to take into account only a suitable distance of probability distributions, this is no longer the case for multistage models. In a sense, this observation seems to illustrate the complexity results obtained in the recent paper [33]. It is shown there that multistage stochastic programs have higher complexity than two-stage models. Techniques for generating and reducing scenario trees in multistage stochastic programs, which are based on stability arguments, have to respect both probability and filtration distances as both contribute to changes of optimal values. Example 2.7 provides estimates for the filtration distance if some scenario is deleted. Since the forward and backward scenario tree generation algorithms developed in [10] and [12] consist of recursive deletions of 
scenarios on increasing or decreasing time horizons $\{1, \ldots, t\}, t=2, \ldots, T$, the estimates derived in Example 2.7 may be extended to tree generation. Such extended estimates may then be used to modify the corresponding algorithms. A discussion of such modifications will be presented in the forthcoming paper [13].

\section{Acknowledgement:}

This work was supported by the DFG Research Center Matheon "Mathematics for key technologies" in Berlin and by a grant of EDF - Electricité de France. The first two authors wish to thank the members of the OSIRIS Division at R\&D of EDF for several stimulating discussions on scenario trees and stability. We extend our gratitude to René Henrion (WIAS Berlin) for his comments on an earlier version of this paper.

\section{References}

[1] Barty, K.: Contributions à la discrétisation des contraintes de mesurabilité pour les problèmes d'optimisation stochastique, Thèse de Doctorat, École Nationale des Ponts et Chaussées, 2004.

[2] Boylan, E.S.: Equiconvergence of martingales, The Annals of Mathematical Statistics 42 (1971), 552-559.

[3] Cotter, K.D., Convergence of information, random variables and noise, Journal of Mathematical Economics 16 (1987), 39-51.

[4] Dupačová, J.; Consigli, G.; Wallace, S. W.: Scenarios for multistage stochastic programs, Annals of Operations Research 100 (2000), 25-53.

[5] Dupačová, J.; Gröwe-Kuska, N.; Römisch, W.: Scenario reduction in stochastic programming: An approach using probability metrics, Mathematical Programming Ser. A 95 (2003), 493-511.

[6] Evstigneev, I.: Measurable selection and dynamic programming, Mathematics of Operations Research 1 (1976) 267-272.

[7] Fiedler, O., Römisch, W.: Stability in multistage stochastic programming, Annals of Operations Research 56 (1995), 79-93.

[8] Fortet, R.; Mourier, E.: Convergence de la répartition empirique vers la répartition théorique, Ann. Sci. Ecole Norm. Sup. 70 (1953), 266-285.

[9] Givens, C.R.; Shortt, R.M.: A class of Wasserstein metrics for probability distributions, Michigan Mathematical Journal 31 (1984), 231-240.

[10] Gröwe-Kuska, N.; Heitsch, H.; Römisch, W.: Scenario reduction and scenario tree construction for power management problems, IEEE Bologna Power Tech Proceedings (A. Borghetti, C.A. Nucci, M. Paolone eds.), 2003 IEEE.

[11] Heitsch, H.; Römisch, W.: Scenario reduction algorithms in stochastic programming, Computational Optimization and Applications 24 (2003), 187-206. 
[12] Heitsch, H.; Römisch, W.: Generation of multivariate scenario trees to model stochasticity in power management, IEEE St. Petersburg Power Tech 2005 (to appear).

[13] Heitsch, H.; Römisch, W.: Scenario tree modelling for multistage stochastic programs, in preparation.

[14] Hochreiter, R., Pflug, G.: Scenario tree generation as a multidimensional facility location problem, AURORA Technical Report, Department of Statistics, University of Vienna, 2002.

[15] Hoover, D.N.: Convergence in distribution and Skorokhod convergence for the general theory of processes, Probability Theory and Related Fields 89 (1991), 239-259.

[16] Kaňková, V.: Empirical estimates in multistage stochastic programs, Report No. 1930, Institute of Information Theory and Automation, Academy of Sciences of the Czech Republic, 1998.

[17] Neveu, J.: Note on the tightness of the metric on the set of complete sub $\sigma$ algebras of a probability space, The Annals of Mathematical Statistics, 43 (1972), 1369-1371.

[18] Olsen, P.: Multistage stochastic programming with recourse as mathematical programming in an $L_{p^{-}}$space, SIAM Journal Control and Optimization 14 (1976), 528-537.

[19] Olsen, P.: Discretizations of multistage stochastic programming problems, Mathematical Programming Study 6 (1976), 111-124.

[20] Pennanen, T.: Epi-convergent discretizations of multistage stochastic programs via integration quadratures, Stochastic Programming E-Print Series 19-2004 (<www.speps.info〉).

[21] Pflug, G. Ch.: Scenario tree generation for multiperiod financial optimization by optimal discretization, Mathematical Programming 89 (2001), 251-271.

[22] Rachev, S. T.: Probability Metrics and the Stability of Stochastic Models, Wiley, 1991.

[23] Rachev, S. T.; Römisch, W.: Quantitative stability in stochastic programming: The method of probability metrics, Mathematics of Operations Research 27 (2002), 792-818.

[24] Rachev, S. T.; Rüschendorf, L.: Mass Transportation Problems, Vol. I and II, Springer, Berlin 1998.

[25] Rachev, S. T.; Schief, A.: On $L_{p}$-minimal metrics, Probability and Mathematical Statistics 13 (1992), 311-320.

[26] Rockafellar, R. T.; Wets, R. J-B: Nonanticipativity and $\mathcal{L}^{1}$-martingales in stochastic optimization problems, Mathematical Programming Study 6 (1976), 170-187.

[27] Rockafellar, R. T.; Wets, R. J-B: Variational Analysis, Springer-Verlag, Berlin, 1998.

[28] Römisch, W.: Stability of Stochastic Programming Problems, in: Stochastic Programming (A. Ruszczyński and A. Shapiro Eds.), Handbooks in Operations Research and Management Science, Volume 10, Elsevier, Amsterdam 2003, 483-554. 
[29] Römisch, W.; Schultz, R.: Stability analysis for stochastic programs, Annals of Operations Research 30 (1991), 241-266.

[30] Rogge, L.: Uniform inequalities for conditional expectations, Annals of Probability 2 (1974), 486-489.

[31] Ruszczyński, A.; Shapiro, A. (Eds.): Stochastic Programming, Handbooks in Operations Research and Management Science, Volume 10, Elsevier, Amsterdam 2003.

[32] Shapiro, A.: Inference of statistical bounds for multistage stochastic programming problems, Math. Meth. Oper. Res. 58 (2003), 57-68.

[33] Shapiro, A.; Nemirovski, A.: On complexity of stochastic programming problems, Eprint available at http://www.optimization-online.org, 2004.

[34] Strugarek, C.: On the Fortet-Mourier metric for the stability of stochastic programming problems, Stochastic Programming E-Print Series 25-2004 (<www.speps.info>). 